general hospitals and specialist hospitals division of responsibilities, organisational structure, two-way referral mechanism and communities to monitor the operation of the network is not quite built the whole, restricted the development of community health services. (2) Lack of dynamic data, management information missing. Community Center, the household as the unit set up a health file, but the health records are now almost become a dead file, and did not track population health problems. Therefore, it cannot reflect the health record of the progress of the disease in patients with hypertension and prevention of, intervention in the situation. (3) Targeted poor health education. The health of residents and living behaviour concepts are outmoded, self-health awareness is poor, the seriousness of the harm of chronic disease awareness rate is low, the risk factors for inadequate understanding of the significance of interventions.

Objectives In response to these problems, the Beijing Chui yangliu hospital under the "Beijing cardiovascular and cerebrovascular diseases prevention and control manual" requirement, proposed "Hypertension in the community-hospital management at different levels between the test mode, "Compliance rate and blood pressure in order to standardise management rate, lower risk levels, to reduce the risk of cardiovascular morbidity and mortality, to explore suitable for China's situation model of the integrated management of hypertension. The study through management at different levels of inclusion of 274 patients with hypertensive patients before and after their own comparative study to evaluate the model after the implementation of the hypertension prevention and control results.

Method Adopt the "Chinese Hypertension Prevention Guide" (2005 revised edition) diagnostic criteria and requirements, in December 2008-2009 years for 274 cases of hypertension management status for analysis.

Results (1) General situation: there were 129 case of men and 145 cases of women in 274 patients with hypertension, the average age of $67.3 \pm 9.4$ years; Educational level is relatively high, secondary and higher educational level accounted for $81.4 \%$ of the total; In the hierarchical management process, with a total of 16 cases were lost, accounting for $5.8 \%$ of the number of standardised management, including those who refuse to measure the number of defaulters accounted for $43.7 \%$. (2) Awareness rate: The management of the knowledge rate were significantly higher than the management of the former, the difference is still statistically significant $(p<0.01)$, Among them, "the daily salt intake and reasonable" and "control high blood pressure measures" the most significant, awareness by management of the former increased by $16.3 \%$ and $27.9 \%$ to $65.5 \%$ and $78.7 \%$; the management of the right to determine the standard blood pressure increased awareness rate $82.6 \%$. The results show that, Management at different levels of behaviour change in patients with hypertension had a greater role, also targeted individual, diversified health education also exposes patients to improve the level of knowledge. (3) Management at different levels by 1 year, patients with bad habits was significantly reduced; The overall management of the rate of $94.2 \%$, which strengthen the management of 123 cases, 114 cases of general management, management, rates were $96.9 \%$ and $91.9 \%$. After the Management of different levels, the results showed that 63 patients of very high risk group $(38.7 \%)$ down to high risk group, 26 patients of high risk group (45.6\%) down to medium risk group, 8 patients of medium risk group $(32.0 \%)$ down to low risk group, 11 patients of low risk group $(42.3 \%)$. In the management level, strengthen the management group, 62 patients with reduced general management group, downgrade rate was $50.4 \%$, moderate management group dropped to nine cases general management group, downgrade rate was $42.9 \%$. After statistical analysis, management, the indicators before and after differences were statistically significant $(p<0.05)$. (4) According to the hierarchical management standards, the number of 258 cases of standardised management, of which 220 cases of blood pressure control effect of good and can still control the rate of $85.3 \%$. (5) 1. Comparison of blood pressure control rate was no significant difference between male and female, $(p>0.05)$. 2. Age effects on blood pressure control rates were significantly different, which the 45-65 age group is better blood pressure control in patients, $(p<0.01)$. Age risk factors in elderly patients is not easy to change their behaviour and lead to more complications associated with the causes of low blood pressure, low control rate of young patients with unhealthy living habits and poorer compliance. 3. Educational level influence on blood pressure control rate, differences among the groups was statistically significant, $(p<0.01)$. High blood pressure education controls are better than low education who, due to the different education level led to their level of awareness of hypertension, attention to different degrees, resulting in the management of compliance and self-management there are differences. 4. Control of body mass index in patients with normal blood pressure within the good level of control in overweight or obese. 5. Related knowledge among the higher, blood pressure control rate. 6 . Better way of life of patients control their blood pressure was higher than those bad habits blood pressure control rate. 7 . Medication affect the situation on the blood pressure control rate, regular medication of patients reached their blood pressure control rate of $70.9 \%$. 8. Management began, dangerous levels of blood pressure control rate among all groups showed no significant difference, $(p<0.01)$. 9. Management-level influence on blood pressure control rate, difference was statistically significant lower levels of management step by step help to improve compliance rates of blood pressure, $(p<0.01)$. (6) Whether control of blood pressure as dependent variable, a single-factor analysis of the factors was significant for the independent variable, logistic regression analysis, the results showed that: factors that affect the model results are BMI, Awareness, Lifestyle, Medication conditions and Management level $(\mathrm{p}<0.01)$.

Conclusions (1) Implementation of the hierarchical management model one year later, which effectively improved hypertension awareness, management and control of hypertension rates. (2) Factors that affect the model results are BMI, Awareness, Lifestyle, Medication conditions and Management level.

\section{E0297 TREATMENT OF STATINS IN SECONDARY PREVENTION OF HIGH RISK CORONARY HEART DISEASE IN CHINESE-BRIG STUDY}

doi:10.1136/hrt.2010.208967.297

Li Hongjuan, Liu Jun, Liu Jing, Wang Wei, Li Yan, Qin Lanping, Wang Miao, Sun Jiayi, Qi Yue, Zhao Dong Department of Epidemiology, Beijing Anzhen Hospital, The Capital University of Medical Science, Beijing Insititute of Heart Lung and Blood Vessel Diseases

Purpose To determine in patients with established coronary heart disease (CHD) whether the guidelines recommendations on secondary prevention of $\mathrm{CHD}$ are being followed.

Methods 64 hospitals across 31 provinces of China, including 32 secondary hospitals and 32 tertiary hospitals were selected for a baseline survey. Fifty outpatients with established acute coronary syndrome (ACS) recruited consecutively in each hospital. Information of these patients was collected and the situation of statins among the patients was analysed.

Results A total of 2516 high risk CHD outpatients were involved in present report. Mean age of the patients was $65 \pm 10$ and $69.4 \%$ was male. Fifty-seven point nine percentages of patients were treated with statins at the moment of interview and $29.8 \%$ had achieved the recommended LDL-cholesterol target. The situation was significant difference among regions and provinces. The South China had the most patients with statins treatment, but the Northeast of China least. Treatment success rate was highest in 
Central China. At this interview, $68.2 \%$ outpatients were prescribed statins and $24.1 \%$ prescribed doses of statins were sub-minimal.

Conclusion There was a gap between Chinese patients with CHD prevention recommended in guidelines and that in the "real world."

\section{e0298 INTENSIVE CHOLESTEROL LOWERING WITH SIMVASTATIN IMPROVES OUTCOMES OF PERCUTANEOUS CORONARY INTERVENTION IN PATIENTS WITH ACUTE CORONARY SYNDROME}

doi:10.1136/hrt.2010.208967.298

Fu Xianghua, Jia Xinwei, Wang Yanbo, Wang Xuechao, Gu Xinshun, Zhang Jing, Hao Guozhen, Jiang. The Second Hospital of Hebei Medical University

Objective To evaluate the immediate protective effects of intensive statin pretreatment on myocardial perfusion and myocardial ischaemic injury during PCI.

Methods A total of 228 acute coronary syndrome (ACS) patients were randomly divided into standard statin group (SSG, $\mathrm{n}=115$ ) and intensive statin group (ISG, $\mathrm{n}=113$ ). Patients in SSG received $20 \mathrm{mg}$ simvastatin and patients in ISG received 80 mgsimvastatin for 7 days before PCI. TIMI grade flow (TGF), corrected TIMI frame count (CTFC) and TIMI myocardial perfusion grade (TMPG) of the intervened vessel were recorded before and after stent deployment. Plasma level of CK-MB and cTnI were measured before and $24 \mathrm{~h}$ after the procedure.

Results The TFG after stent deployment was significantly improved with less TIMI $0-1$ patients and more TIMI 3 blood flow in ISG than in SSG (all $p<0.05)$. Patients with no reflow phenomenon were less in ISG $(p<0.001)$. The CTFC was lower in ISG than SSG $(p<0.001)$. TMPG was also improved in ISG than SSG $(p=0.001)$. Twenty-four hours after the procedure, although PCI caused significantly increase in CK-MB, the elevated CK-MB value was lower in ISG than SSG $(18.74 \pm 8.41$ vs $21.78 \pm 10.64 \mathrm{p}=0.018)$. Similar changes were also found with regard to Troponin I $(0.99 \pm 1.07$ vs $1.47 \pm 1.54, p=0.006)$. No myocardial infarction was found. Among them, myocardial necrosis was detected in $13 \%$ of the patients in SSG, while $4.4 \%$ in ISG $(p=0.021)$. Myocardial infarction was found in $4.4 \%$ in the patients in SSG and $0.9 \%$ in ISG $(\mathrm{p}=0.213)$.

Conclusion Intensive statin pretreatment for 7 days before PCI can further improve myocardial blood perfusion, protect myocardium from ischaemic injury.

\section{e0299 INCIDENCE AND PREDICTORS OF HYPERTENSION AMONG RURAL CHINESE ADULTS: RESULTS FROM LIAONING PROVINCE}

doi:10.1136/hrt.2010.208967.299

Sun Zhaoging, Sun Yingxian. Shengjing Hospital of China Medical University

Objective To determine the incidence of hypertension and its risk factors among rural Chinese adults.

Methods A population-based sample of 24360 rural Chinese adults aged $\geq 35$ years and free from hypertension at baseline were followed from 2004-2006 to 2008. Incident hypertension was defined as systolic pressure $\geq 140 \mathrm{~mm} \mathrm{Hg}$, diastolic pressure $\geq 90 \mathrm{~mm} \mathrm{Hg}$, or current use of antihypertensive medication.

Results Over a mean of 28 months of follow-up, $29.6 \%$ of men and $23.4 \%$ of women developed hypertension. The age-adjusted incidence rate was higher in men (12.75 per 100 person-years) than in women (10.04 per 100 person-years). Among men, independent predictors of incident hypertension were baseline age $[R R$ per 5 years: 1.11 ; $95 \%$ CI 1.10 to 1.13 ], Mongolian ethnicity (RR: 1.09;
95\% CI 1.01 to 1.18 ), use of alcohol, (RR: $1.14 ; 95 \%$ CI 1.06 to 1.23 ), high income versus low income (RR: $1.11 ; 95 \%$ CI 1.00 to 1.22 ; RR: $1.11 ; 95 \%$ CI 1.03 to 1.20 ), prehypertension versus normotension (RR: 1.18; 95\% CI 1.08 to 1.28), overweight and obesity (RR: 1.28; $95 \%$ CI 1.17 to 1.40 ), baseline salt intake (RR per one g/day: $1.00 ; 95 \%$ CI 1.00 to 1.01 ) and family history of hypertension (RR: 1.14; $95 \%$ CI 1.03 to 1.27). With the exception of use of alcohol, mean income, low physical activity was shown as risk factors in women; the results were similar for women. The awareness, treatment and control rates for newly developed hypertension were 29.9\%, 19.5\% and $1.5 \%$ respectively.

Conclusions These data indicate that the incidence of hypertension is high among these rural Chinese adults and it is associated with many risk factors. And suggest that most newly developed hypertension cases are not treated. These increases in hypertension are probably related to rapid social changes in our country and may apply to other areas of the developing world. These results call for urgent improvements in hypertension prevention and control programs in rural China.

\section{Epidemiology and Preventive Medicine: Lipid Research \\ E0300 RELATIONSHIP OF POLYMORPHISM OF APOLIPOPROTEIN E ALLELES WITH CORONARY HEART DISEASE}

doi:10.1136/hrt.2010.208967.300

Zou Yangchun, Hu Dayi, Tong Qiguang, Xu Liujian, Jia Xingyuan. Beijing Meitan General Hospital

Objective To study the relationship of the apolipoprotein (apo) E with coronary heart disease (CHD).

Methods We studied 68 patients with CHD and 59 control subjects without CHD. Polymorphism of ApoE alleles were measured by PCR, Hhal enzyme digestion and polyacrylamide gel electrophoresis. Results TC, TG, LDL-c, apoB, LP (a) level of CHD group were higher than control $(p<0.05)$; There were significant differences in gene frequency of apo E alleles between CHD and control group but HDL, ApoA1 level of CHD group were lower than control; when compared between the $\mathrm{E} 3$ and the $\mathrm{E} 4$, the $\mathrm{E} 2$ frequency were related with $\mathrm{CHD}(\mathrm{RR}=3.5611,95 \%$ CI 1.1693 to 6.8963, $\mathrm{p}<0.01)$.

Conclusion ApoE gene polymorphisms affects serum TC, LDL-C, apoB and LP (a) levels in CHD and apoE allele E2 is a risk factor in the occurrence of CHD.

\section{e0301 THE RELATIONSHIP BETWEEN APOLIPOPROTEIN E GENE POLYMORPHISMS AND CORONARY ATHEROSCLEROSIS DISEASE IN THE NORTHERN CHINESE}

doi:10.1136/hrt.2010.208967.301

Zou Yangchun, Hu Dayi, Tong Oiguang, Xu Liujian, Jia Xingyuan. Beijing Meitan General Hospital

Background Previous studies on the attribution of apolipoprotein (apo) E polymorphisms on the occurrence of coronary atherosclerosis disease (CAD) showed inconsistent results. Apo $\mathrm{E}$ is a constituent of lipoproteins with considerable variation due to cysteine-arginine exchanges. We investigated the relation between apo E gene polymorphism and the occurrence of $\mathrm{CAD}$ in northern Chinese.

Methods The distributions of the HhaI polymorphisms of the apo $\mathrm{E}$ gene and blood lipids levels were determined among 132 Chinese subjects in relation to circulating lipids and coronary angiography. 